\title{
Analysis of the Structure-stability Relationship of Cold-adapted Lipase PsLip1 from Homology Modeling
}

\section{Dongwon Choo*}

Department of Bioinformatics, Korea Bio-Polytechnic, Nonsan 320-905, Korea

\begin{abstract}
Two initial models of cold-adapted lipase PsLip1 have been constructed, based on homology with the bacterial lipases Chromobacterium viscosum (CvLip) and Pseudomonas cepacia (PcLip), whose X-ray structures have been solved and refined to high resolution. The mature polypeptide chains of these lipases have $84 \%$ similarity. The models of Mod1 and Mod2 have been compared with the tertiary structures of CvLip and PcLip, respectively, and analyzed in terms of stabilizing interactions. Several structural aspects that are believed to contribute to protein stability have been compared: the number of conserved salt bridges, aromatic interactions, hydrogen bonds, helix capping, and disulfide bridges. The 3-dimensional structural model of PsLip1 has been constructed in order to elucidate the structural reasons for the decreased thermostability of the enzyme in comparison with its mesophilic counterparts.
\end{abstract}

Keywords: cold-adapted lipase, thermostability, homology modeling

\section{Introduction}

Microorganisms living in extreme environments have adapted to their habitants in such a way that metabolic processes permit them to survive and function. Among these adaptations are: specific mechanisms for energy transduction and for the regulation of the intracellular environment and metabolism, stability of structural and functional components of the cell membrane and cell wall, reaction kinetics, protein conformation, and function of enzymes (Atlas and Bartha, 1987; Prins et al., 1990; Suzuki et al., 2003; Tutino et al., 2009). One limiting factor for survival is temperature. All microorganisms have a characteristic optimal growth temperature at which they exhibit their highest growth and reproductive

${ }^{*}$ Corresponding author: E-mail dwchoo@kopo.ac.kr Tel +82-41-746-7371, Fax +82-41-746-7370 Accepted 17 May 2011 rate and a range of temperatures that they can tolerate.

Psychrotrophic microorganisms constitute a major class in the microbial world, which has vast regions of permanently cold environments: deep-sea water and polar and alpine regions. In spite of the diversity and abundance of these extremophiles, the numerous physiological and biochemical adaptations to life at temperatures close to $0^{\circ} \mathrm{C}$ remain poorly documented (Joseph, 2008; Morita, 1975; Russel, 1992).

The analysis of the structure-stability relationship of proteins from extremophiles and the comparison with their mesophilic counterparts have provided insights into the factors governing thermal stability. Several structures of mesophilic enzymes have been solved and compared with their mesophilic counterparts (Fujinaga et al., 1993; Hennig et al., 1995; Korndorfer et al., 1995; Russel et al., 1994; Yip et al., 1995). So far, at the other end of the spectrum, enzymes from psychrophilic organisms, has been largely neglected. This is due mainly to the instability of psychrophilic enzymes and, in some cases, the lack of isogony to their mesophilic counterparts. Sequence comparison and structure prediction have been performed on the cold-adapted lipase PsLip1 from the psychrotrophic bacteria Alaskan Pseudomonas sp. B11-1. A 3-dimensional structural model for PsLip1, based on the structures of lipases, has thus far clarified. the structural reasons for the decreased thermostability of the enzyme in comparison with its mesophilic counterparts.

\section{Methods}

\section{DNA sequencing}

All methods concerning DNA manipulation were performed as described by Sambrook et al. (1989). All bases were sequenced at least once in each direction using the chain termination method with Dye Primer Terminator sequencing kits, using an Applied Biosystem 370A DNA sequencer. Sequence analysis was carried out with the software program DNASTAR (DNASTAR, Inc., USA).

\section{Molecular Modeling}

Multiple sequence alignment was carried out by using the MEGALIGN program of the DNASTAR package by Clustal method. The PHD secondary structure prediction and prediction-based threading methods (Rost, B. and 
Sander, C., 1993) were used through electronic mail at http://www.emblheidelberg.de/predictprotein/ppDoPred.html. Atomic coordinates were obtained from the Brookhaven Protein DATA Base for CvLip (code CVL) and PcLip (code 2LIP). Two initial models were constructed by homology modeling from CVL coordinates as a reference structure (Mod1) and from the 2LIP coordinates as a reference structure (Mod2) by the Automated Knowledgebased Protein Modeling Server (Swiss-Model) at the GLAXO Institute for Molecular Biology in Geneva, Switzerland (GIMB) (Peitsch et al., 1996) via the internet (http://expasy.Hcuge.ch/swissmod/SWISS-MODEL.html). The quality of these structures has been examined by the PROCHECK (Laskowski et al., 1993) and WHAT IF (Vriend, G., 1990) programs of the Biotech Validations Suite for Protein Structures (EMBL Heidelberg, Germany) via the internet (http://biotech.embl-heidelberg.de:8400/). The initial models were refined by energy minimization using the steepest descent method, followed by the conjugate gradient method. The minimization calculations were conducted using the CHARM module of the QUANTA software package (QUANTA 4.0; Molecular Simulations, Burlington, MA). The final models were first evaluated using the Protein Health module of QUANTA and then by 3D profile method (Bowie et al., 1991), as described by Luthy et al. (1992). The calculations were performed on Silicon Graphics Indigo2 or Unix systems.

\section{Results and Discussion}

PsLip1 showed maximal activity at $35^{\circ} \mathrm{C}$ toward p-nitrophenyl laurate. The enzyme was found to be stable at $30^{\circ} \mathrm{C}$ : less than $10 \%$ of the original activity was lost in $60 \mathrm{~min}$. However, nearly $90 \%$ of the activity was lost at $60^{\circ} \mathrm{C}$ in $60 \mathrm{~min}$ and completely lost at $70^{\circ} \mathrm{C}$ in $60 \mathrm{~min}$. The activation energy for the hydrolysis of p-nitrophenyl laurate was determined to be $12 \mathrm{kcal} / \mathrm{mol}$ in the range of $5^{\circ} \mathrm{C}$ to $35^{\circ} \mathrm{C}$. The activation energies for enzymes from other sources have been reported with the same substrate: Antarctic bacteria, $12-17 \mathrm{kcal} / \mathrm{mol}$; mesophilic Pseudomonas, aeruginosa, $25 \mathrm{kcal} / \mathrm{mol}$ (Feller et al., 1990).

Two initial models of PsLip1 have been constructed, based on its homology with two bacterial lipases, Chromobacterium visccosum (CvLip) and Pseudomonas cepacia (PcLip), whose X-ray structures have been solved and refined to high resolution. The mature polypeptide chains of these lipases have $84 \%$ similarity. The PsLip1 protein sequence was aligned to both the PcLip and CvLip sequences with the bl2seq program on NCBI. The PsLip1 sequence shows a sequence identity of $47 \%$ and $32 \%$ to lipases from PcLip and CvLip, respectively. However, there are large and significant differences in the tertiary structures of the enzymes due to different conformations, based on the differences in
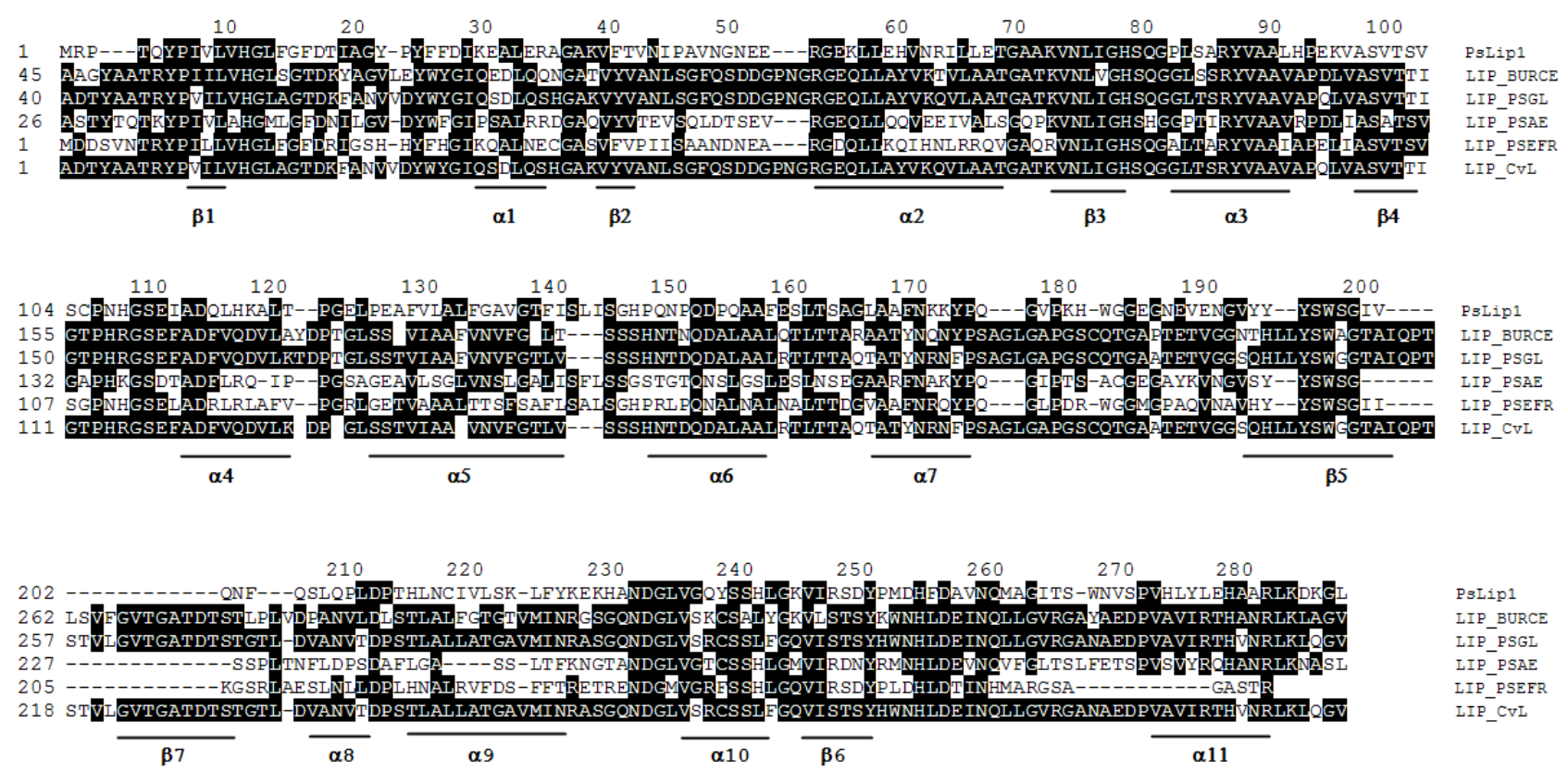

Fig. 1. Sequence alignment of PsLIp1, five lipases from Pseudomonas sp., and Chromobacterium viscosum. The alignment was carried out with the CLUSTAL V program of DNASTAR (DNASTAR, Inc., USA). The solid boxes show the deletion sequences of PsLip1 compared with other lipases. The secondary structure elements are given for the Chromobacterium viscosum lipase. 
crystallization conditions. In the CvLip structure, the catalytic triad is not exposed to the solvent, whereas in PcLip, the active site is highly open to the solvent through the opening at the helical lid (Kim et al., 1997; Lang et al., 1996).

The initial model of PsLip1 (Mod1-closed conformation) was constructed based on reference structure of CvLip by using Optimist mode of Swiss-Model, which allows customization of the performed modeling by user defined sequences and reference structure. Initially, five homologous lipase sequences were aligned by Clustal method (Fig. 1). Then, two lipases (PsF and PsA) were deleted, and the necessary corrections have been introduced manually, based on PHD secondary structure prediction and prediction-based threading (Rost et al., 1993) (Fig. 2).

Several possible sequence alignments have been used as input files for Swiss-Model. The model with the best stereochemical properties was used further for refinement by energy minimization. The same procedure has been used for construction of the second model of
PsLip1 (Mod2 open conformation) but was based on the reference structure of PcLip.

The calculation of close contacts revealed no large aromatic overlaps left in the final models of PsLip1. The last evaluation of the quality of the models was performed by 3D profile analysis. The profile window plots of reference structures and final models agreed well. The Luthy/Einsenberg score for Mod1 was 79.0 (84.7 for the X-ray structure of CvLip) and 69.5 for Mod2 (85.8 for the X-ray structure of PcLip). However the reliability of the models was low in the refined structure before insertion (L143-S145) and long deletion (I200-P209).

\section{Analysis of the models and relation with thermo- stability}

The Mod1 and Mod2 models have been compared with the tertiary structures of CvLip and PcLip, respectively, and analyzed in terms of stability of the contribution to protein-stabilizing interactions. Several structural aspects that are believed to contribute to protein stability have

----MRPTOYPIVLVHGLFGFDTIAGYP--YFFDIKEALERAGAKVFTVNIPAV- -NGNEFRGEKLLEHVNRILLET GAA -ADNYAATRYPI ILVHGLTGTDKYAGVLEYWYGIQEDLQQRGATVYVANL SGFQS DDGPNGRGEQLLAYVKTVLAAT GAT -ADTYAATRYPVILVHGLAGTDKF ANVVDYWYGIQSDLQSHGAKVYVANL SGFQS DDGP NGRGEQLLAYVKQVLAAT GAT $\beta 1$ $\alpha 1 \quad \beta 2$ $\alpha 2$

$82 \quad 102 \quad 122 \quad 142 \quad 162$
KVNLIGHSQGPLSARYVAALHPEKVASVTSVSCPNHGSEIADQLHKAL--TPGEL PEAFVLALFGAVGTFISL ISGH PQN KVNLVGHSQGGLTSRYVAAVAPDLVASVTTIGT PHRGSEFADFVQGVLAYDPTGLSSSVIAAFVNVFGILTS---SSNNT KVNLIGHSQGGLTSRYVAAVAPQLVASVTTIGT PHRGSEFADFVQDVLKT DPTGLSSTVIAAFVNVFGTLVS---SSHNT $\beta 3$ $\alpha 3$ $\beta 4$ $\alpha 4$ $\alpha 5$

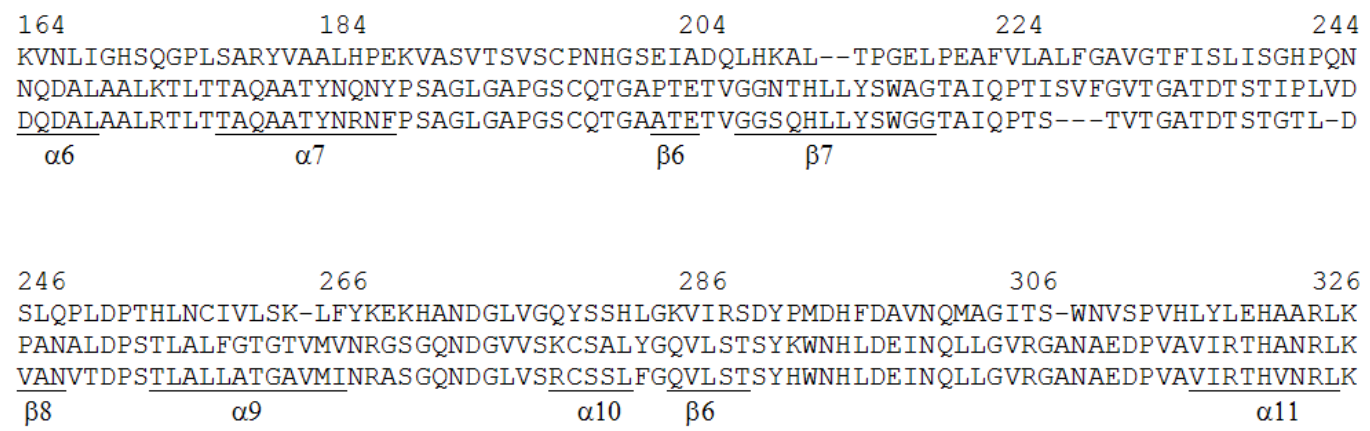

$\begin{array}{ll}327 & \\ \text { DKGL } & \text { PsLip1 } \\ \text { LAGV } & \text { 12Lip.pdb } \\ \text { LQGV } & \text { 11CVL.pdb }\end{array}$

Fig. 2. Amino acid sequence alignment by Swiss-Model serve. Corrections have introduced manually, based on PHD secondary structure prediction and prediction-based threading. PsLip1, CvLip, and CvLip indicate Pseudomonas sp. B11-1., Pseudomonas cepacia lipase, and Chromobacterium viscosum lipase, respectively. 
Table 1. Parameters possibly responsible for thermostability

\begin{tabular}{|c|c|c|c|c|c|}
\hline \multirow{2}{*}{ Parameter } & \multicolumn{2}{|c|}{ Models of PsLip1 } & \multicolumn{2}{|c|}{$\begin{array}{l}\text { Reference } \\
\text { structures }\end{array}$} & \multirow{2}{*}{$\begin{array}{c}\text { Effects o } \\
\text { PsLip1 } \\
\text { stability }\end{array}$} \\
\hline & Mod I & Mod II & CvLip & PcLip & \\
\hline Salt bridges & 12 & 11 & 9 & 8 & + \\
\hline $\begin{array}{l}\text { Aromatic } \\
\text { interactions }\end{array}$ & 8 & 8 & 11 & 8 & - \\
\hline Hydrogen bonds $\mathrm{s}^{\mathrm{a})}$ & $265 / 0.92$ & $249 / 0.87$ & $347 / 1.1$ & $324 / 1.01$ & - \\
\hline $\begin{array}{l}\text { Helix capping } \\
\text { N-caps/C-caps }\end{array}$ & $5 / 2$ & $4 / 2$ & $5 / 2$ & $4 / 1$ & 0 \\
\hline Loop insertions & 1 & 1 & 8 & 8 & + \\
\hline Disulfide bridges & 0 & 0 & 1 & 1 & - \\
\hline Proline contents & 18 & 18 & 10 & 13 & + \\
\hline Arg/(Arg + Lys) & 0.32 & 0.32 & 0.65 & 0.56 & - \\
\hline
\end{tabular}

a)Total numbers of hydrogen bonds and normalization to length of polypeptide chain.

been compared: the number of conserved salt bridges, aromatic interactions, hydrogen bonds, helix capping, and disulfide bridges (Table 1).

\section{(1) Salt bridges}

The electrostatic interactions between side chains of charged residues are believed to contribute essentially to protein stability. A salt bridge is defined as an ion pair with a distance of 2.5-4.0 $\AA$ between charged non-hydrogen atoms. Comparisons between 3-dimensional structures of thermophilic, mesophilic, and psychrophilic enzymes (homology-based models) indicate a clear correlation between increasing numbers of potential salt bridges and enzyme themostability. In the case of PsLip1, the amount of potential salt bridges is similar to that of mesophilic enzymes and even slightly higher.

\section{(2) Aromatic-aromatic interactions}

These interactions were defined as pairs of aromatic residues with distances between the phenyl ring centroids of less than $7 \AA$. In the case of PsLip1, both models have the same amount of such interactions as the open structure of PcLip, in which a large hydrophobic area and active center are exposed to the solvent. Four residues of the aromatic cluster in the core of the structure of CvLip (F122-F119-F142-F146) are disrupted in PcLip1 and absent in both models of PsLip1. The decreased amount of aromatic-aromatic interactions in the core of Mod1 of PsLip1 in comparison with the corresponding structure of CvLip could mostly contribute to the decreased thermostability of the psychrotrophic protein.

\section{(3) Hydrogen bonds}

The hydrogen bonds shown in Table 1 were calculated using a distance cut-off of $3.3 \AA$ and an angle cut-off of $90^{\circ}$, with the linear hydrogen bond 0 . The distance cut-off was applied to the distance between the hydrogen donor and acceptor atoms. The angle formed by the acceptors, hydrogen, and donor atoms was subjected to the cut-off. Because the lengths of the polypeptide chains of these three lipases are different, the numbers of hydrogen bonds were also normalized by the number of residues. The total, as well as normalized, numbers of hydrogen bonds were larger in the case of mesophilic enzymes as compared with the psychrotrophic one. It is likely that the hydrogen bonds play a significant role in the enhanced thermostability of mesophilic enzymes.

\section{(4) Helix capping}

The $\mathrm{N}$ - and C-terminal of an $\alpha$-helix have unsatisfied main chain hydrogen bonds. The side chains of the first ( $\mathrm{N}$-cap) and the last ( $\mathrm{C}$-cap) residues of an $\alpha$-helix may be able to provide the necessary hydrogen bonding partners or stabilize the helix by electrostatic interactions with the helix dipole. The characteristics of the helix caps in PsLip1 in terms of their stabilizing influence are similar to those found in other enzymes.

\section{(5) Loop insertions}

Loops are believed to be important in protein stability, as they might provide initiation points for unfolding. However, PsLip1 has only a single three-residue insertion as compared with eight insertions of different sizes in mesophilic enzymes. The 216-234 region of the longest insertion for mesophilic enzymes (which is absent in PsLip1) is rather flexible, such that three residues, 220-222, could not be seen in the CvLip structure. It seems likely that insertions in mesophilic enzymes will destabilize the structure as compared with PsLip1.

\section{(6) Disulfide bridges}

Introduction of disulfide bridges could significantly contribute to protein stability. PsLip1 contains two cysteine residues, $105 \mathrm{C}$ and $217 \mathrm{C}$. However, the distances between the $\mathrm{C} \alpha$ and $\mathrm{S}$ atoms (15.6 and 16.6 $\AA$, respectively) are too great to form a disulfide bridge. The absence of disulfide bonds in PsLip1 as compared with mesophilic lipases containing one intact disulfide bond could contribute to the decreased thermostability of psychrotrophic enzymes.

\section{(7) Proline contents}

The pyrrolidon ring of proline is believed to decrease the backbone entropy of unfolding by decreasing the main-chain flexibility. Unexpectedly, the number of proline residues in PsLip1 is larger than that in its mesophilic counterparts, with 18 (PsLip1), 13 (PcLip), and 10 (CvLip) prolines residues, respectively. In fact, this is the largest number of proline residues found throughout the 5 compared sequences. It seems that the additional proline residues could contribute to the stabilization of 
PsLIp1.

\section{(8) $\mathrm{Arg} /($ Arg + Lys) ratio}

The molar ratio of basic residues Arg/(Arg + Lys) is equal to 0.32 , which is low with respect to the values for CvLip (0.65) and PcLip (0.56). The stabilizing effect of arginine has the ability to form more stable intermolecular bonds than lysine. Moreover, arginine interacts more favorably with the solvent than lysine at the protein-water interface. It seems that the number of stabilizing residues, such as arginine, is reduced in coldadapted lipase, which possibly has a flexible tertiary structure.

The author built a model for the 3D structure of PsLip1 from Pseudomonas sp. B11-1, based on alignments of known structures. The 3D structures of $P$. g/umae, $P$. cepacia, and $C$. viscosum lipases have been solved. The requirement for a conformational change in PsLip1 in order to allow access to the active site has been confirmed by calculation and examination of the solvent-accessible surface. The orientation demonstrates that a repositioning of $\alpha 5$ upon activation would allow the cavities around the active site, thus allowing access of the substrate to the active site.

A 3D model for PsLip1 has been analyzed for possible structural reasons for the decreased thermostability of the enzyme compared with its mesophilic counterparts. As shown for other proteins isolated from organisms that are adapted to grow at low temperature, a reduction in aromatic-aromatic interactions, a smaller number of hydrogen bonds, and reduced Arg/(Arg + Lys) ratio as well as the absence of disulfide bridges could contribute to decreased thermostability. Two homologous lipase structures from mesophilic organisms (CVL-CvLip and 2LIP-PcLip) have been used for homology model construction. The possible structural reasons for the decreased thermostability of psychrotrophic enzymes in comparison with its mesophilic counterparts could be a reduction in aromatic-aromatic interactions, reduced Arg/(Arg+Lys) ratio, a smaller number of hydrogen bonds, and the absence of a disulfide bridge. However, PsLip1 has more proline residues and shorter surface loops, which could be regarded as stabilizing factors. The contribution of salt bridges to thermostability seems to be insignificant in this particular case. This study may provide insight into the factors governing thermal stability through an analysis of the structure-stability relationship of proteins from extrophiles and a comparison with their mesophilic counterparts.

\section{References}

Atlas, R.M. and Bartha, R. (1987). In Microbial Ecology. Fundamentals and Applications, 2nd edition, (Menlo Park,
CK: Benjamin Cummings Publ. Comp. Inc), pp.233-262.

Feller, G., Thiry, M., Aspigny, J.L., Mergeay, M., and Gerday, C. (1990). Lipases from psychrotropic antarctic bacteria. FEMS Microbiol. Lett. 66, 239-244.

Fujinaga, M., Berthed-Colominas, C., Yaremchuck, A.D., Yukalo, M.A., and Cusack, S. (1993). Refined crystal structure of the seryl-tRNA synthetase from Thermus thermophilus at $2.5 \mathrm{~A}$ resolution. J. Mol. Biol. 234, 222-233.

Hennig, M., Darimont, B., Sterner, R., Kirschner, K., and Jansonius, J.N. (1995). $2.0 \AA$ structure of indole-3-glycerol phosphate synthase from the hyperthermophile Sulfolobus solfataricus: possible determinants of protein stability. Structure 3, 1295-1306.

Joseph B., Ramteke P.W., and Thomas G. (2008). Cold active microbial lipases: some hot issues and recent developments. Biotechnol. Adv. 26, 457-470.

Kim, K.K., Song, H.K., Shin, D.H., Hwang, K.Y., and Suh, S.W. (1997). The crystal structure of a triacylglycerol lipase from Pseudomonas cepacia reveals a highly open conformation in the absence of a bound inhibitor. Structure 5, 173-185.

Korndorfer, I., Steipe, B., Huber, R., Tomschy, A., and Jaenicke, R. (1995). The crystal structure of holo-glyceraldehyde-3-phosphate dehydrogenase from the hyperthermophilic bacterium Thermotoga maritima at $2.5 \AA$ resolution. J. Mol. Biol. 246, 511-521.

Lang, D., Hoffmann, B., Haalck, L., Hecht, H.J., Spenner, F., Shimid, R.D., and Schmburg, D. (1996). Crystal structure of a bacterial lipase from Chromobacterium viscosum ATCC 6918 refined at 1.6 angstroms resolution. J. Mol. Biol. 259, 704-717.

Laskowski, R.A., MacArthur, M.W., Moss, D.S., and Thornton, J.M. (1993). PROCHECK: a program to check the stereochemical quality of protein structures. J. Appl. Cryst. 26, 283-291.

Luthy , R., Bowie, J.U., and Einsenberg, D. (1992). Assessment of protein models with three-dimensional profiles. Nature 356, 83-85.

Morita, R.Y. (1975). Psychrophilic bacteria. Bacterial. Rev. 39, 144-167.

Prins, R.A., de Vriji, W., Gottschal, J.C., and Hansen, T.A. (1990). Adaptation of microorganisms to extreme environments. FEMS Microbiol. Rev. 75, 103-104.

Rost, B. and Sander, C. (1993). Prediction of protein secondary structure at better than $70 \%$ accuracy. J. Mol. Biol. 232, 584-599.

Russel, N.J. (1992). In Molecular biology and biotechnology of extremophiles. Herbert, R.A. and Sharp, R.J., eds. (Blackie), pp.203-224.

Russel, R.J.M., Hough, D.W., Danson, M.J., and Taylor, G.L. (1994). The crystal structure of citrate synthase from the thermophilic archaeon, Thermoplasma acidophilum. Structure 2, 1157-1167.

Sambrook, J., Fritsch, E.F., and Maniatis, T.C. (1989). Molecular cloning (New York: Cold Spring Harber Laboratory Press).

Suzuki, T., Nakayama, T., Choo, D.W., Hirano, Y., Kurihara, T., Nishino, T., and Esaki, N. (2003). Cloning, heterolo- 
84 Genomics \& Informatics Vol. 9(2) 79-84, June 2011

gous expression, renaturation, and characterization of a cold adapted esterase with unique primary structure from a psychrotroph Pseudomonas sp. strain B11-1. Protein Expr. Purif. 30, 171-178.

Tutino, M.L., di Prisco, G., Marino, G., and de Pascale, D. (2009). Cold-adapted esterase and lipase: from fundmentals to application. Protein Pept. Lett. 16, 1172-1180.

Vriend, G. (1990). WHAT IF: a molecular modeling and drug design program. J. Mol. Graph. 8, 52-56.

Yip, K.S.P., Stillman, T.J., Britton, K.L., Artymiuk, P.J., Baker, P.J., Sedelnikova, S.E., Engel, P.C., Pasqua, A., Chiaraluce, R., Consalvi, V., Scandurra, R., and Rice, D.W. (1995). The structure of Pyrococcus furiosus glutamate dehydrogenase reveals a key role for ion pair networks in maintaining enzyme stability at extreme temperatures. Structure 3, 1147-1158. 